\title{
Unsere französischen Kollegen diskutieren die Zukunft der Medizin
}

\section{Jean Martin}

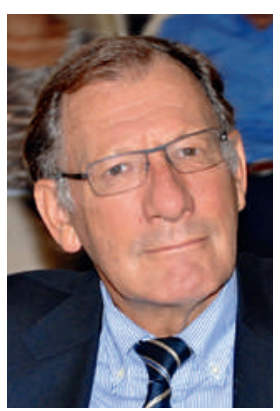

1 Nr. 37, Nov.-Dez. 2014, S. 12-15. Ordre des médecins, 180 bd Haussmann F-75389 Paris Cedex o8.

2 Schweizerische Akademie der Medizinischen Wissenschaften. Studie der SAMW «Haltung der Ärzteschaft zur Suizidhilfe». Schweiz Ärztezeitung. 2014;95(47):1767-9.
Im Oktober 2014 veranstaltete die französische Ärztegesellschaft (Ordre national - eine öffentlich-rechtliche Einrichtung, nicht nur ein Verband wie die FMH) 70 Jahre nach ihrer Gründung ihren ersten Kongress. Grundlage der Veranstaltung: «Ein Gefühl der Erschöpfung, der mangelnden Anerkennung seitens staatlicher Stellen, aber auch seitens der Gesellschaft. Die Ärzteschaft sieht sich seit mehreren Jahren stigmatisiert, vor immer neuen Zwängen, wo sie doch zu den nationalen Reichtümern zählt!» (So die Zeitschrift der Kammer mit dem Namen médecins) [1].

Dazu Dr. Bouet, Präsident der "Ordre»: «Wir werden nicht als fundamentales Regulativ für potentielles Leiden von Gesellschaft und Individuum wertgeschätzt. Ärzte engagieren sich voll für die Verbesserung der Lebensumstände.» Ein anderer Teilnehmer: «Wenn der einzelne Mitbürger gehalten ist, die Gesellschaft ein Stück voranzubringen, so ist es der Arzt noch umso mehr.»

Partnerschaft. Feststellung eines Gastes, des Soziologen Maffesoli: «Wir funktionieren nicht mehr auf der Vertikalität des Wissenden (Experten) mit einer edukativen Haltung im Sinne von «ich weiss, du nicht〉. Es gibt eine Art neuer Sozialisierung. Der medizinische Bezug ist ein Prozess des Begleitens und Teilens.» Der Präsident: «Es gibt weder die ideale Gesellschaft, noch den idealen Arzt.» Der Arzt von heute darf nicht davon ausgehen, dass er allein alles Wissen hat. Er muss vielmehr in der Lage sein, dieses Wissen zu nutzen. Patienten sind verantwortliche Personen, die wir an all unseren Überlegungen und unserem Handeln teilhaben lassen müssen.»

Komplexität. Dr. Rachel Bocher, Vorsitzende einer Spitalärzte-Gewerkschaft: «Die dem Gesundheitssystem inhärente Komplexität, seine politischen Konsequenzen, soziokulturellen und wirtschaftlichen Unterschiede machen deutlich, warum es - angesichts der grossen Veränderungen und Innovationen unserer Zeit - so schwierig ist, ein universelles und gerechtes System zu entwerfen.» Dazu Michel Maffesoli: «Verstand ist nicht mehr alles, es gibt eine Rückkehr zur Emotion. Es gilt, menschliche Parameter zu integrieren, zu experimentieren ... Dabei soll der Terminus «experimentieren` nicht abschrecken! Das Leben verläuft oft nicht gradlinig. Wir können nicht alles be- herrschen. Die Schwierigkeit besteht darin, dass wir uns der Realität anpassen müssen.»

Ein Statut und spezielle Rollen. "Menschen brauchen Mittler, die ihnen helfen, Entgangenes zu verstehen und die sie bei sozialen, familiären und umweltspezifischen Problemen unterstützen. «Einen Arzt zu konsultieren, erscheint nicht nur als Zuflucht, sondern vielmehr als ultimative Freiheit> (Dr. Bocher). Meine (J.M.) Erfahrung hat mich überzeugt, dass die Ärzteschaft mehrerlei Funktionen zu erfüllen hat. Zum einen ist sie zuständig für die Gesundheit der Patienten, zum anderen ist sie aber auch Mittler, Verteidiger von Rechten und übt eine gewisse moralische Autorität aus, auch in der Funktion des 'Whistleblowers) angesichts strittiger Entwicklungen (wie jüngst beim Thema der ärztlichen Schweigepflicht in Gefängnissen).

Es bleiben Differenzen. U.a. bei den am Ende eines Lebens anstehenden Fragen. Der dem französischen Staatspräsidenten von Prof. Sicard im Dezember 2012 vorgelegte Bericht unterstrich zahlreiche, sehr unbefriedigende Situationen im Land, darunter u.a. die Schwierigkeit, am Ende eines Lebens das strikte Recht des Patienten anzuerkennen, Pflege zurückzuweisen oder historische Vorbehalte gegenüber einer ausreichenden Schmerzbehandlung. In der oben genannten Zeitung gibt es einen kurzen Vermerk darüber, dass Vertreter der "Ordre" am 15. Oktober 2014 eine parlamentarische Kommission zum Thema Lebens ende getroffen haben. Sie legten zehn Prinzipien vor, denen sie sich verpflichtet fühlt, beginnend mit der strikten Weigerung, den Tod vorsätzlich herbeizuführen oder ihn über eine Suizidbeihilfe zu ermöglichen. Eine Divergenz zu den jüngsten Ergebnissen der von der SAMW in Auftrag gegebenen Studie [2], in der 3/4 aller Schweizer Ärzte antworten, dass sie die Sterbehilfe akzeptieren. Ein vergleichbarer Teil der Bevölkerung tut dies übrigens ebenfalls.

Zwar mag die französische Ärzteschaft nicht unberechtigt als konservativ gelten, doch die Ideen entwickeln sich weiter, auch in der Ärztegesellschaft selbst, die sich sozial und gesellschaftlich verpflichtet fühlt. Die vorrangige Aufgabe des Arztes ist der Dienst am Patienten. Dieser wiederum ist unauflöslich mit dem Dienst an der Gesundheit der Gemeinschaft verknüpft. Mir scheint, als könnten wir uns in vielen Punkten der Meinung unserer französischen Kolleginnen und Kollegen anschliessen. 